Supporting Information

\title{
Microcalorimetric and Conductivity studies with Micelles prepared from Multi-headed Pyridinium Surfactants
}

\author{
Santanu Bhattacharya, and Jayanta Haldar
}

Table of Contents:

Scheme S1

Synthesis 


\section{Scheme S1 ${ }^{\mathrm{a}}$}

A.

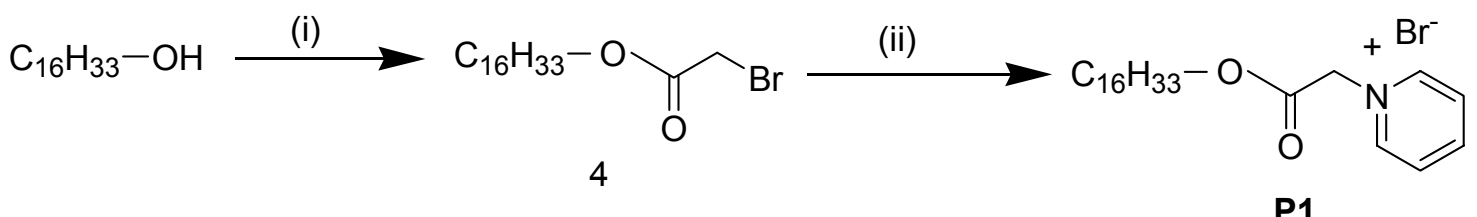

B.

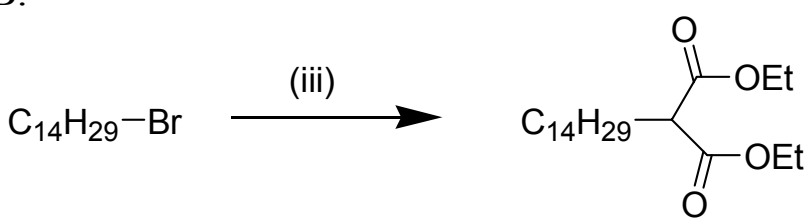

5

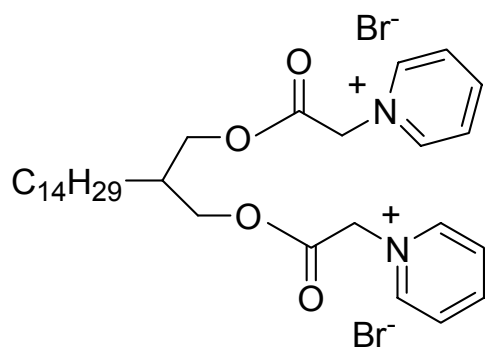

P1

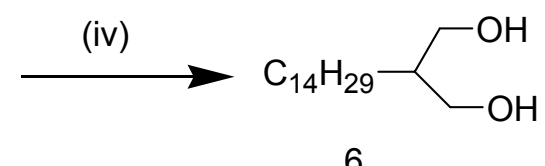

6<smiles>CCCCCCCCC(COC(=O)CBr)COC(=O)CBr</smiles>

P2

C.

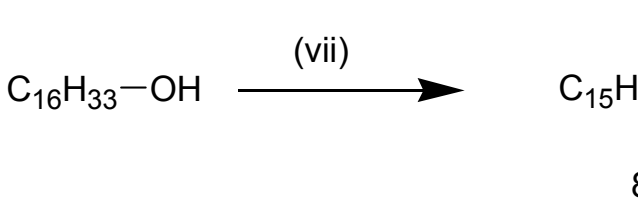

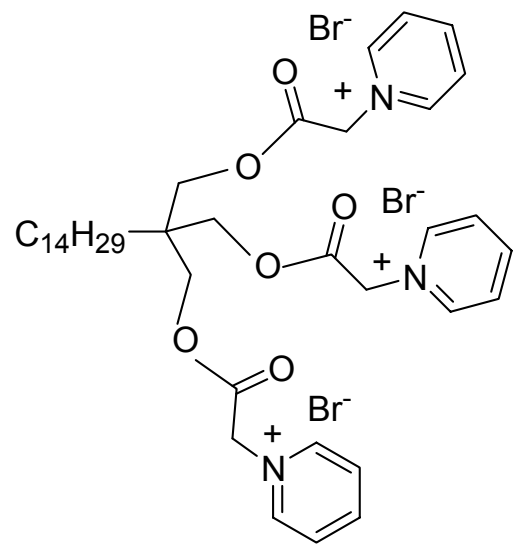

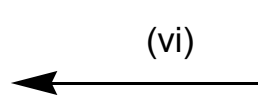

7

P3
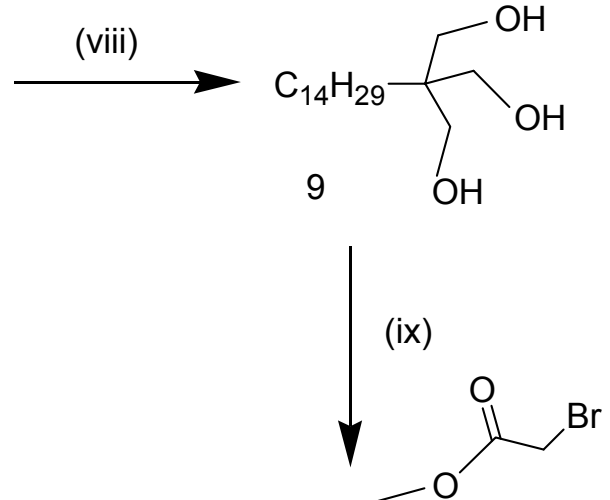

${ }^{a}$ Reagents, conditions and yields: (i) $\mathrm{BrCH}_{2} \mathrm{COBr}, \mathrm{DMAP}, \mathrm{Et}_{3} \mathrm{~N}, \mathrm{CH}_{2} \mathrm{Cl}_{2}, 0{ }^{\circ} \mathrm{C}(10 \mathrm{~min})$ and rt (1 h), 91\%; (ii) Pyridine, acetone, pressure tube, $50{ }^{\circ} \mathrm{C}$ (12 h), 90\%.(iii) Diethyl 
malonate, $\mathrm{NaH}$, THF, $0{ }^{\circ} \mathrm{C}(100 \mathrm{~min})$, rt $(2 \mathrm{~h})$, and $70{ }^{\circ} \mathrm{C}(15 \mathrm{~h}), 71 \%$; (iv) $\mathrm{LiAlH}_{4}, \mathrm{AlCl}_{3}$, $\mathrm{Et}_{2} \mathrm{O}, 0{ }^{\circ} \mathrm{C}(20 \mathrm{~min})$ and $\mathrm{rt}(1 \mathrm{~h}), 96 \%$; (v) $\mathrm{BrCH}_{2} \mathrm{COBr}$, DMAP, $\mathrm{Et}_{3} \mathrm{~N}, \mathrm{CH}_{2} \mathrm{Cl}_{2}, 0{ }^{\circ} \mathrm{C}(10$ min) and rt (3 h), 64\%; (vi) Pyridine, acetone, pressure tube, $50{ }^{\circ} \mathrm{C}(24 \mathrm{~h}), 78 \%$. (vii) PCC, $\mathrm{CH}_{2} \mathrm{Cl}_{2}$, rt (5 h), 95\%; (viii) formaldehyde solution, $\mathrm{KOH}, 50 \%$ aqueous $\mathrm{EtOH}$, rt (4 h) and $50{ }^{\circ} \mathrm{C}(2 \mathrm{~h}), 41 \%$; (ix) $\mathrm{BrCH}_{2} \mathrm{COBr}$, DMAP, $\mathrm{Et}_{3} \mathrm{~N}, \mathrm{CH}_{2} \mathrm{Cl}_{2}, 0{ }^{\circ} \mathrm{C}$ (10 min) and rt (5 h), 53\%; (x) Pyridine, acetone, pressure tube, $50{ }^{\circ} \mathrm{C}(48 \mathrm{~h}), 62 \%$.

\section{Experimental Section}

Materials. All the starting materials were obtained from Aldrich or Fluka and used as received. Pyridinium chlorochromate (PCC) was synthesized as described by Corey et al. ${ }^{1}$ CPB used in this study were purchased from Aldrich. All the solvents were reagent grade and dried prior to use. The surfactants were synthesized as described in scheme S1. Column chromatography was performed using 60-120 mesh silica gel. NMR spectra were recorded using Jeol JNM $\lambda-300\left(300 \mathrm{MHz}\right.$ for $\left.{ }^{1} \mathrm{H}\right)$ or Bruker AMX-400 $\left(400 \mathrm{MHz}\right.$ for ${ }^{1} \mathrm{H}$ and $133.3 \mathrm{MHz}$ for ${ }^{13} \mathrm{C}$ ) spectrometer. The chemical shifts $(\delta)$ are reported in ppm downfield from the internal standard; TMS, for ${ }^{1} \mathrm{H}-\mathrm{NMR}$ and ${ }^{13} \mathrm{C}-\mathrm{NMR}$. Mass spectra were recorded on a Kratos PCKompact SEQ V1.2.2 MALDI-TOF spectrometer or on a MicroMass ESI-TOF spectrometer or Shimadzu table-top GC-MS or ESI-MS (HP1100LC-MSD). Infrared (IR) spectra were recorded on a Jasco FT-IR 410 spectrometer using $\mathrm{KBr}$ pellets or neat. The surfactant CPB (99\% Fluka, USA) was used

after recrystallization using $\mathrm{CHCl}_{3} /$ ethyl acetate mixture. Doubly distilled water (specific conductivity 2-4 $\mu \mathrm{Scm}^{-1}$ at $313 \mathrm{~K}$ ) was used to prepare the solutions for both conductometric and calorimetric studies. 


\section{Synthesis.}

Hexadecyl bromoethanoate, (4). 1-Hexadecanol (1.0 g, $4.13 \mathrm{mmol})$ was dissolved in dry $\mathrm{CH}_{2} \mathrm{Cl}_{2}(8 \mathrm{ml})$. DMAP $(0.068 \mathrm{~g}, 0.56 \mathrm{mmol})$ and dry $\mathrm{Et}_{3} \mathrm{~N}(0.74 \mathrm{ml})$ were added to the reaction mixture and cooled in ice-water bath. Bromoacetyl bromide $(0.43 \mathrm{ml}, 4.95$ mmol) was added drop wise into the reaction mixture and after $10 \mathrm{~min}$ the ice-bath was removed and it was allowed to stir for $1 \mathrm{~h}$ at room temperature. The reaction mixture was diluted with $\mathrm{CHCl}_{3}(50 \mathrm{ml})$ and successively washed with dilute $\mathrm{HCl}$ solution $(2 \mathrm{M}, 10$ $\mathrm{ml})$ and with water $(10 \mathrm{ml})$. Finally it was washed with $\mathrm{NaHCO}_{3}(0.5 \mathrm{M}, 10 \mathrm{ml})$ and saturated brine solution $(20 \mathrm{ml})$. The organic layer was separated and dried over anhydrous $\mathrm{Na}_{2} \mathrm{SO}_{4}$, and concentrated to afford a residue, which was purified by column chromatography over silica gel using EtOAc/hexane (1:99) solvent mixture. A low melting, white compound ( $\mathrm{R}_{\mathrm{f}} \sim 0.8$ in EtOAc/hexane (1:99) solvent mixture) was isolated in $91 \%$ yield. FT-IR (neat): $1740 \mathrm{~cm}^{-1}$ (C=O str.). ${ }^{1} \mathrm{H}-\mathrm{NMR}\left(\mathrm{CDCl}_{3}, 300 \mathrm{MHz}\right): \delta: 0.88$ ( $t$, terminal $\left.\underline{\mathrm{CH}}_{3}, 3 \mathrm{H}\right), 1.25$ (br $\left.m, \underline{\mathrm{CH}}_{2} \times 13,26 \mathrm{H}\right), 1.59-1.7\left(m, \mathrm{C}_{2} \mathrm{CH}_{2} \mathrm{O}, 2 \mathrm{H}\right), 3.85$ (s, $\left.\mathrm{OC}(\mathrm{O}) \underline{\mathrm{C}}_{2}, 2 \mathrm{H}\right), 4.17\left(t,-\mathrm{CH}_{2} \underline{\mathrm{C}}_{2} \mathrm{O}-, 2 \mathrm{H}\right)$. MALDI-TOF: $m / z=386.4[\mathrm{M}+\mathrm{Na}]^{+}$.

Hexadecyl- $N$-ethanoate pyridinium bromide, P1. Hexadecyl bromoethanoate (4) (1.45 g, $3.99 \mathrm{mmol})$ was dissolved in dry acetone $(10 \mathrm{ml})$ and taken in a screw top pressure tube. Dry pyridine $(0.96 \mathrm{ml}, 11.97 \mathrm{mmol})$ was added in the pressure tube and the mixture was heated at $50{ }^{\circ} \mathrm{C}$ for $12 \mathrm{~h}$. A white solid was formed which was filtered and washed repeatedly with dry acetone. Pure surfactant P1 (1.59 g, 90\%) was obtained upon several recrystallizations from $\mathrm{CHCl}_{3} / n$-hexane. FT-IR (KBr): $1748 \mathrm{~cm}^{-1}\left(\mathrm{C}=\mathrm{O}\right.$ str.). ${ }^{1} \mathrm{H}-$ NMR (300 MHz, $\left.\mathrm{CDCl}_{3}\right): \delta: 0.88\left(t\right.$, terminal $\left.\mathrm{C}_{3}, 3 \mathrm{H}\right), 1.25\left(\right.$ br $\left.m,\left(\mathrm{C}_{2}\right)_{13}, 26 \mathrm{H}\right), 1.64-$ $1.69\left(m, \underline{\mathrm{C}}_{2} \mathrm{CH}_{2} \mathrm{O}, 2 \mathrm{H}\right), 4.21\left(t, \mathrm{C}_{2} \mathrm{O}, 2 \mathrm{H}\right), 6.3\left(s, \mathrm{COC}_{2}, 2 \mathrm{H}\right), 8.08(t, m-\underline{\mathrm{H}}, 2 \mathrm{H}), 8.53$ 
$(t, p-\underline{\mathrm{H}}, 1 \mathrm{H}), 9.42(d, J=6.6 \mathrm{~Hz}, o-\underline{\mathrm{H}}, 2 \mathrm{H}) .{ }^{13} \mathrm{C}-\mathrm{NMR}\left(133.3 \mathrm{MHz}, \mathrm{CDCl}_{3}\right): \delta: 14.07$, $22.65,25.68,28.40,29.34,29.50,29.69,31.91,61.28,127.75,146.11,146.78,165.81$. ESI-MS: $m / z=\mathrm{M}^{+}\left[\mathrm{C}_{23} \mathrm{H}_{40} \mathrm{NO}_{2}\right]^{+}$: calcd: 362.5 , found: 362.5. Elemental analysis: (\%) for $\mathrm{C}_{23} \mathrm{H}_{40} \mathrm{NO}_{2} \mathrm{Br}$ : calcd: C 62.4, H 9.1, N 3.16; found: C 62.2, H 9.2, N 3.05.

Ethyl-2-carboethoxyhexadecanoate, (5). $\mathrm{NaH}(1.25 \mathrm{~g}, 26 \mathrm{mmol}, 50 \%$ dispersion in oil) was taken in a two-necked round bottom flask closed with septum to which dry $n$ hexane $(5 \mathrm{ml})$ was added and stirred for $5 \mathrm{~min}$. to dissolve the mineral oil. The supernatant in $n$-hexane was removed by a syringe, dry THF $(10 \mathrm{ml})$ was added and stirred at $0{ }^{\circ} \mathrm{C}$ for $15 \mathrm{~min}$. Diethyl malonate $(5.66 \mathrm{~g}, 35.3 \mathrm{mmol})$ in dry THF $(10 \mathrm{ml})$ was then slowly added for $30 \mathrm{~min}$ at $0{ }^{\circ} \mathrm{C}$ to the resulting suspension. The solution became clear after $10 \mathrm{~min}$ of stirring. The stirring was continued for another $30 \mathrm{~min}$. 1Bromotetradecane (4 g, $14.4 \mathrm{mmol})$ in dry THF (15 ml) was added drop wise over $30 \mathrm{~min}$ to the stirred solution, resulting in the formation of a white viscous residue which was diluted with dry THF $(10 \mathrm{ml})$ and allowed to stir at room temperature for $2 \mathrm{~h}$, followed by refluxing for $15 \mathrm{~h}$ at $70{ }^{\circ} \mathrm{C}$ to ensure the completion of the reaction. The mixture was then taken up in $\mathrm{CHCl}_{3}(200 \mathrm{ml})$ and washed first with water $(100 \mathrm{ml} \times 3)$ and then with $10 \%$ $(\mathrm{w} / \mathrm{v})$ brine solution $(150 \mathrm{ml})$. The $\mathrm{CHCl}_{3}$ layer was separated and dried using anhydrous $\mathrm{Na}_{2} \mathrm{SO}_{4}$. The solvent was evaporated to leave a gum like residue which was adsorbed on silica gel and the required compound $\left(\mathrm{R}_{\mathrm{f}} \sim 0.6\right.$ in EtOAc/hexane (4:96) solvent mixture) was purified by column chromatography. Pure compound was isolated in $71 \%$ (3.6 g) yield. FT-IR (neat): $1735 \mathrm{~cm}^{-1}\left(\mathrm{C}=\mathrm{O}\right.$ str.). ${ }^{1} \mathrm{H}-\mathrm{NMR}\left(300 \mathrm{MHz}, \mathrm{CDCl}_{3}\right): \delta: 0.88(t$, terminal $\left.\mathrm{C}_{3}, 3 \mathrm{H}\right), 1.2-1.3\left(\right.$ br $\left.m,\left(-\mathrm{C}_{2}\right)_{12},-\mathrm{OCH}_{2} \underline{\mathrm{C}}_{3} \times 2,30 \mathrm{H}\right), 1.8\left(m,-\underline{\mathrm{C}}_{2} \mathrm{CH}-, 2 \mathrm{H}\right)$, $3.3\left(t,-\mathrm{CH}_{2} \mathrm{C} \underline{\mathrm{H}}-, 1 \mathrm{H}\right), 4.15\left(q,-\mathrm{OC}_{2} \mathrm{CH}_{3} \times 2,4 \mathrm{H}\right)$. LR-MS: $m / z=357[\mathrm{M}+\mathrm{H}]^{+}$. 
2-Hydroxymethyl hexadecanol, (6). Anhydrous $\mathrm{AlCl}_{3}(0.634 \mathrm{~g}, 4.76 \mathrm{mmol})$ was added slowly to a solution of $\mathrm{LiAlH}_{4}(0.639 \mathrm{~g}, 16.8 \mathrm{mmol})$ in dry diethyl ether $(20 \mathrm{ml})$ at $0{ }^{\circ} \mathrm{C}$. The color of the mixture became white, to which Ethyl-2-carboethoxy hexadecanoate, (5) $(1 \mathrm{~g}, 2.8 \mathrm{mmol})$ in the form of a solution in dry diethyl ether $(10 \mathrm{ml})$ was added drop wise. The reaction mixture was allowed to stir at $0{ }^{\circ} \mathrm{C}$ for $20 \mathrm{~min}$ and then at room temperature for $1 \mathrm{~h}$. At the end of this period, moist $\mathrm{Et}_{2} \mathrm{O}(10 \mathrm{ml})$ was added slowly and stirred for $10 \mathrm{~min}$. The reaction mixture was then washed with $\mathrm{HCl}$ solution $(2 \mathrm{~N}, 10 \mathrm{ml})$ and the aqueous layer was extracted with EtOAc $(50 \mathrm{ml} \times 2)$. All the organic layers were obtained and dried over anhydrous $\mathrm{Na}_{2} \mathrm{SO}_{4}$ and the solvent was evaporated to leave a white solid, which was separated by column chromatography using silica gel. A white solid was obtained $(0.73 \mathrm{~g}, 96 \%)$ towards the end. FT-IR (nujol): $3300 \mathrm{~cm}^{-1}(\mathrm{O}-\mathrm{H}$ str.). ${ }^{1} \mathrm{H}-\mathrm{NMR}\left(300 \mathrm{MHz}, \mathrm{CDCl}_{3}\right): \delta: 0.85\left(t\right.$, terminal $\left.\mathrm{C}_{3}, 3 \mathrm{H}\right), 1.23$ (br $m,\left(-\underline{\mathrm{C}}_{2}-\right)_{13}$, $26 \mathrm{H}), 1.7-1.8\left(m,-\mathrm{CHCH}_{2^{-}}, 1 \mathrm{H}\right), 3.6-3.8\left(m, \mathrm{CHC}_{2} \mathrm{OH} \times 2,4 \mathrm{H}\right)$. LR-MS: $m / z=273$ $[\mathrm{M}+\mathrm{H}]^{+}$.

2-(Methyl bromoethanoate) hexadecyl bromoethanoate, (7). 4- $N, N$-dimethylamino pyridine (DMAP) $(0.05 \mathrm{~g}, 0.41 \mathrm{mmol})$ and $\mathrm{Et}_{3} \mathrm{~N}(0.53 \mathrm{ml}, 3.82 \mathrm{mmol})$ were added to 2hydroxymethyl hexadecanol, (6) $(0.4 \mathrm{~g}, 1.47 \mathrm{mmol})$ dissolved in $\mathrm{CH}_{2} \mathrm{Cl}_{2}(6.0 \mathrm{ml})$, which was cooled at $0{ }^{\circ} \mathrm{C}$. Bromoacetyl bromide $(0.3 \mathrm{ml}, 3.52 \mathrm{mmol})$ was then added drop wise at this temperature. After $\sim 10$ min the ice-bath was removed and the stirring was continued at room temperature for $3 \mathrm{~h}$. The solvent from reaction mixture was evaporated and the required compound was purified by column chromatography over silica gel using EtOAc/hexane (4:96) solvent mixture $\left(\mathrm{R}_{\mathrm{f}} \sim 0.5\right.$ in EtOAc/hexane (6:94) solvent mixture). A low melting white solid was obtained (0.48 g, 64\%). FT-IR (neat): $1743 \mathrm{~cm}^{-1}$ and 1730 
$\mathrm{cm}^{-1}(\mathrm{C}=\mathrm{O}$ str. $) .{ }^{1} \mathrm{H}-\mathrm{NMR}\left(300 \mathrm{MHz}, \mathrm{CDCl}_{3}\right): \delta: 0.88\left(t\right.$, terminal $\left.\mathrm{C}_{3}, 3 \mathrm{H}\right), 1.25$ (br $m$, $\left.\left(-\underline{\mathrm{C}}_{2}-\right)_{13}, 26 \mathrm{H}\right), 2.06-2.17\left(m, \mathrm{C}_{\mathrm{HCH}} \mathrm{O}, 1 \mathrm{H}\right), 3.85\left(s,-\mathrm{OC}(\mathrm{O}) \underline{\mathrm{H}}_{2} \times 2,4 \mathrm{H}\right), 4.15\left(d d, J_{1}\right.$ $\left.=11.25 \mathrm{~Hz}, J_{2}=6.3 \mathrm{~Hz},-\mathrm{CH}\left(\mathrm{CH}_{\mathrm{a}} \mathrm{H}_{\mathrm{b}} \mathrm{O}-\right)_{2}, 2 \mathrm{H}\right), 4.22\left(d d, J_{1}=11.1 \mathrm{~Hz}, J_{2}=5.4 \mathrm{~Hz},-\right.$ $\left.\mathrm{CH}\left(\mathrm{CH}_{a} \underline{\mathrm{H}}_{b} \mathrm{O}-\right)_{2}, 2 \mathrm{H}\right)$. MALDI-TOF: $m / z=537.8[\mathrm{M}+\mathrm{Na}]^{+}$.

\section{2-(Methyl- $N$-ethanoate pyridinium bromide) hexadecyl- $N$-ethanoate-pyridinium}

bromide, P2. Inside a screw-top pressure tube, 2-(methyl bromoethanoate) hexadecyl bromoethanoate (7) $(0.77 \mathrm{~g}, 1.49 \mathrm{mmol})$ was dissolved in dry acetone $(8 \mathrm{ml})$. Dry pyridine $(0.72 \mathrm{ml}, 8.94 \mathrm{mmol})$ was added in the pressure tube and it was heated at $50{ }^{\circ} \mathrm{C}$ for $24 \mathrm{~h}$. A gummy solid was formed on the wall of the pressure tube. $\mathrm{CHCl}_{3}$ was added to the reaction mixture and it was transferred into a round bottom flask. The solvent and extra pyridine was removed under vacuum. The required compound, P2 (78 \%) was purified by repetitive precipitations by dissolving the solid with 1:1 mixture of $\mathrm{CHCl}_{3} /$ acetone and then upon drop wise addition of $n$-hexane. The compound was found to be hygroscopic solid. FT-IR ( $\mathrm{KBr}): 1750 \mathrm{~cm}^{-1}$ (C = O str.). ${ }^{1} \mathrm{H}-\mathrm{NMR}(300 \mathrm{MHz}$, $\left.\mathrm{CDCl}_{3}\right): \delta: 0.88\left(t\right.$, terminal $\left.\underline{\mathrm{C}}_{3}, 3 \mathrm{H}\right), 1.26\left(\mathrm{br} m,\left(\mathrm{C}_{2}\right)_{13}, 26 \mathrm{H}\right), 2.02-2.17\left(m, \mathrm{C}_{\mathrm{HCH}} \mathrm{O}\right.$, $1 \mathrm{H}), 4.15\left(d d, J_{1}=7.2 \mathrm{~Hz}, J_{2}=10.8 \mathrm{~Hz},-\mathrm{CHC}^{\mathrm{Ha}} \mathrm{H}_{\mathrm{b}} \mathrm{O}-\times 2,2 \mathrm{H}\right), 4.37\left(d d, J_{1}=3.3 \mathrm{~Hz}, J_{2}\right.$ $\left.=10.8 \mathrm{~Hz},-\mathrm{CHCH}_{a} \underline{\mathrm{H}}_{\mathrm{b}} \mathrm{O}-\times 2,2 \mathrm{H}\right), 6.43\left(A B q, J=17.4 \mathrm{~Hz},{ }^{+}{ }^{\mathrm{NC}} \underline{H}_{a} \underline{\mathrm{H}}_{\mathrm{b}} \times 2,4 \mathrm{H}\right), 8.12(t$, $m-\underline{\mathrm{H}}, 4 \mathrm{H}), 8.59(\mathrm{t}, p-\underline{\mathrm{H}}, 2 \mathrm{H}), 9.55(d, J=6 \mathrm{~Hz}, o-\underline{\mathrm{H}}, 4 \mathrm{H}) .{ }^{13} \mathrm{C}-\mathrm{NMR}\left(133.3 \mathrm{MHz}, \mathrm{CDCl}_{3}\right)$ : $\delta: 14.07,22.68,27.145,27.305,27.90,28.08,29.34,29.71,29.94,31.93,39.82,42.24$, $61.56,61.78,65.86,67.45,128.035,146.36,146.765,165.97$. ESI-MS: $\mathrm{m} / \mathrm{z}=$ $\mathrm{M}^{2+}\left[\mathrm{C}_{31} \mathrm{H}_{48} \mathrm{~N}_{2} \mathrm{O}_{4}\right]^{2+} \mathrm{Br}^{-}$(78.91): calcd: 591.3, found: 591.5, $\mathrm{M}^{2+} \mathrm{Br}^{-}$(80.91): calcd: 593.3, found: 593.5, $\mathrm{M}^{2+} / 2$ : calcd: 256.2, found: 256.2. Elemental analysis: (\%) for $\mathrm{C}_{31} \mathrm{H}_{48} \mathrm{~N}_{2} \mathrm{O}_{4} \mathrm{Br}_{2}, \mathrm{H}_{2} \mathrm{O}$ : calcd: C 53.9, H 7.2, N 4.1; found: C 53.8, H 7.0, N 3.9. 
1-Hexadecanal, (8). ${ }^{2}$ Pyridinium chlorochromate (PCC) (6.68 g, 30.99 mmol) was taken in dry $\mathrm{CH}_{2} \mathrm{Cl}_{2}(100 \mathrm{ml})$ and stirred at room temperature for $5 \mathrm{~min}$. After addition of hexadecanol (5 g, $20.66 \mathrm{mmol})$ to the reaction mixture, it was again allowed to stir for $3 \mathrm{~h}$ at room temperature. The reaction mixture was diluted with dry ether and the upper portion of the solvent was decanted. The insoluble residue was washed 3-4 times with dry ether and the solvent from all the washings along with main $\mathrm{Et}_{2} \mathrm{O}$ fraction was evaporated to have a solid residue. The compound from this residue was purified by column chromatography over silica gel using EtOAc/hexane (2:98) solvent mixture $\left(\mathrm{R}_{\mathrm{f}} \sim 0.7\right.$ in EtOAc/hexane (2:98) solvent mixture). A white solid compound (4.68 g, $95 \%$ ) was obtained. FT-IR (nujol): $1720 \mathrm{~cm}^{-1}$ (C = O str.), $3370 \mathrm{~cm}^{-1}$ (C-H ald. str.). ${ }^{1} \mathrm{H}-\mathrm{NMR}$ (300 $\left.\mathrm{MHz}, \mathrm{CDCl}_{3}\right): \delta: 0.85\left(t\right.$, terminal $\left.\underline{\mathrm{C}}_{3}, 3 \mathrm{H}\right), 1.23-1.26\left(\right.$ br $\left.m, \underline{\mathrm{C}}_{2} \times 13,26 \mathrm{H}\right), 2.36-2.42$ (m, $\underline{\mathrm{C}}_{2} \mathrm{CHO}, 2 \mathrm{H}$ ), 9.74 (br $\left.s, \mathrm{CH}_{2} \mathrm{CHO}, 1 \mathrm{H}\right)$. LR-MS: $m / z=241[\mathrm{M}+\mathrm{H}]^{+}$.

2,2-Bis (hydroxymethyl) hexadecanol, (9). ${ }^{7}$ To a solution of 1-hexadecanal (4.09 g, $17.04 \mathrm{mmol})$, formaldehyde solution $(3.54 \mathrm{ml}, 1.0 \mathrm{ml}$ contains $1.075 \mathrm{~g}$ ) and $50 \%$ aqueous ethanol $(50 \mathrm{ml})$ were added and cooled at $0{ }^{\circ} \mathrm{C} . \mathrm{KOH}(0.954 \mathrm{~g}, 17.04 \mathrm{mmol})$ dissolved in $50 \%$ aqueous ethanol $(50 \mathrm{ml})$ was added to the reaction mixture drop wise. The reaction mixture was allowed to stir at room temperature for $4 \mathrm{~h}$ after which it was heated at $50{ }^{\circ} \mathrm{C}$ for $2 \mathrm{~h}$. Ethanol was evaporated and the reaction mixture was extracted with $\mathrm{Et}_{2} \mathrm{O}$ for 4-5 times. The organic layers were collected together and dried over anhydrous $\mathrm{Na}_{2} \mathrm{SO}_{4}$. A colorless gum like material was obtained after evaporation of the solvent. The required compound $\left(\mathrm{R}_{\mathrm{f}} \sim 0.3\right.$ in 3:2 EtOAc/hexane was purified by column chromatography using silica gel. A white solid compound (2.1 g, 41\%) was isolated. FT-IR (nujol): $3340 \mathrm{~cm}^{-1}$. ${ }^{1} \mathrm{H}-\mathrm{NMR}\left(300 \mathrm{MHz}, \mathrm{CDCl}_{3}\right): \delta: 0.85\left(t\right.$, terminal $\left.\mathrm{C}_{3}, 3 \mathrm{H}\right), 1.15-1.22\left(\mathrm{br} m, \underline{\mathrm{C}}_{2} \times 13\right.$, 
26H), $2.43\left(\right.$ br $\left.\left.s, \mathrm{C}\left(\mathrm{CH}_{2} \mathrm{OH}\right)_{3}, 3 \mathrm{H}\right), 3.72\left(s, \mathrm{C}_{\left(\mathrm{C}_{2}\right.} \mathrm{OH}\right)_{3}, 6 \mathrm{H}\right)$. LR-MS: $m / z=303[\mathrm{M}+$ $\mathrm{H}]^{+}$.

2,2-Bis(methyl bromoethanoate) hexadecyl bromoethanoate (10). 2,2-Bis (hydroxylmethyl) hexadecanol, (9) (0.302 g, $1.22 \mathrm{mmol}$ ) was dissolved in $\mathrm{CH}_{2} \mathrm{Cl}_{2}(7.0$ ml). DMAP $(0.089 \mathrm{~g}, 0.73 \mathrm{mmol})$ and $\mathrm{Et}_{3} \mathrm{~N}(0.66 \mathrm{ml}, 4.76 \mathrm{mmol})$ were added and the reaction mixture was cooled at $0{ }^{\circ} \mathrm{C}$. Bromoacetyl bromide $(0.38 \mathrm{ml}, 4.4 \mathrm{mmol})$ was then added drop wise at this temperature. The ice-bath was removed after $10 \mathrm{~min}$, and the reaction mixture was allowed to stir at room temperature for $5 \mathrm{~h}$. The reaction mixture was diluted with $\mathrm{CHCl}_{3}(30 \mathrm{ml})$ and successively washed with dilute $\mathrm{HCl}$ solution (2 $\mathrm{M}$, $10 \mathrm{ml})$ and with water $(10 \mathrm{ml})$. Finally it was washed carefully with $\mathrm{NaHCO}_{3}(0.5 \mathrm{M}, 10$ ml) and saturated brine solution. The organic layer was separated and dried over anhydrous $\mathrm{Na}_{2} \mathrm{SO}_{4}$. Desired product was isolated by column chromatography over silica gel using EtOAc-hexane solvent mixture. A colorless liquid $\left(\mathrm{R}_{\mathrm{f}} \sim 0.4\right.$ in EtOAc/hexane (8:92) solvent mixture) was obtained $(0.43 \mathrm{~g}, 53 \%)$. FT-IR (neat): $1741 \mathrm{~cm}^{-1}$ (broad C $=\mathrm{O}$ str.). ${ }^{1} \mathrm{H}-\mathrm{NMR}\left(300 \mathrm{MHz}, \mathrm{CDCl}_{3}\right): \delta: 0.88\left(t\right.$, terminal $\left.\mathrm{C}_{3}, 3 \mathrm{H}\right), 1.26\left(\right.$ br $m,\left(-\underline{\mathrm{C}}_{2}-\right)_{12}$, $24 \mathrm{H}), 1.47$ (br $\left.m, \mathrm{C}_{2} \mathrm{C}, 2 \mathrm{H}\right), 3.85\left(s,\left(\mathrm{COC} \underline{H}_{2}\right)_{3}, 6 \mathrm{H}\right), 4.2\left(s,\left(\mathrm{C}_{2} \mathrm{O}\right)_{3}, 6 \mathrm{H}\right)$. MALDITOF: $m / z=689.7[\mathrm{M}+\mathrm{H}]^{+}$.

\section{2,2-Bis (methyl- $N$-ethanoate-pyridinium bromide) hexadecyl- $N$-ethanoate}

pyridinium bromide, P3. 2,2-Bis (methyl bromoethanoate) hexadecyl bromoethanoate, (10) $(0.07 \mathrm{~g}, 0.105 \mathrm{mmol})$ was dissolved in dry acetone $(4 \mathrm{ml})$ and taken in a screw-top pressure tube. Dry pyridine $(1 \mathrm{ml})$ was added in and the mixture was heated at $50{ }^{\circ} \mathrm{C}$ for $48 \mathrm{~h}$. A brown solid was formed on the wall of the pressure tube. The reaction mixture was cooled at room temperature and solvent was decanted carefully from the pressure 
tube. The solid residue was dissolved in $\mathrm{CHCl}_{3}$ and precipitated upon addition of $n$ hexane several times. During the process of precipitation the compound initially came out of the solution as a dense yellow liquid. The solvent could be separated from this by careful decantation to afford a sticky solid material P3 (62\%). The compound was found to be extremely hygroscopic solid. FT-IR ( $\mathrm{KBr}): 1750 \mathrm{~cm}^{-1}$ (C = O str.). ${ }^{1} \mathrm{H}-\mathrm{NMR}(300$ $\left.\mathrm{MHz}, \mathrm{CDCl}_{3}\right): \delta: 0.86\left(t\right.$, terminal $\left.\underline{\mathrm{H}}_{3}, 3 \mathrm{H}\right), 1.26\left(\right.$ br $\left.m,\left(\mathrm{C}_{2}\right)_{13}, 26 \mathrm{H}\right), 4.26\left(s,-\underline{\mathrm{C}}_{2} \mathrm{O}-\times\right.$ $3,6 \mathrm{H}), 6.51\left(s,-\mathrm{COCH}_{2}-\times 3,6 \mathrm{H}\right), 8.09(t, m-\underline{\mathrm{H}}, 6 \mathrm{H}), 8.56(\mathrm{t}, p-\underline{\mathrm{H}}, 3 \mathrm{H}), 9.75(d, J=5.4$ $\mathrm{Hz}, o-\underline{\mathrm{H}}, 6 \mathrm{H}) .{ }^{13} \mathrm{C}-\mathrm{NMR}\left(133.3 \mathrm{MHz}, \mathrm{CDCl}_{3}\right): \delta: 14.09,22.71,27.16,27.32,27.91$, $28.09,29.36,29.72,29.96,31.94,39.84,42.26,61.57,61.79,65.87,67.46,128.04$, 146.37, 146.79, 165.99. ESI-MS: $m / z=\mathrm{M}^{3+}\left[\mathrm{C}_{39} \mathrm{H}_{56} \mathrm{~N}_{3} \mathrm{O}_{6}\right]^{3+} 2 \mathrm{Br}^{-}$(78.91): calcd: 820.7, found: 820.6, $\mathrm{M}^{3+} \mathrm{Br}^{-}$(80.91) $\mathrm{Br}^{-}$(78.91): calcd: 822.6, found: 822.3, $\mathrm{M}^{3+} 2 \mathrm{Br}^{-}$(80.91): calcd: 824.7, found: 824.2, $\mathrm{M}^{3+} \mathrm{Br}^{-}$(78.91)/2: calcd: 370.8, found: $370.8, \mathrm{M}^{3+} \mathrm{Br}^{-}$ (80.91)/2: calcd: 371.8 , found: $371.6, \mathrm{M}^{3+} / 3$ : calcd: 220.9 , found: 220.9 . Elemental analysis: (\%) for $\mathrm{C}_{39} \mathrm{H}_{56} \mathrm{~N}_{3} \mathrm{O}_{6} \mathrm{Br}_{3}, 3 \mathrm{H}_{2} \mathrm{O}$ : calcd: $\mathrm{C} 48.9, \mathrm{H} 6.5, \mathrm{~N}$ 4.4; found: $\mathrm{C} 48.7, \mathrm{H}$ $6.4, \mathrm{~N} 4.5$.

\section{References.}

(1) Corey, E. J.; Suggs, J. W. Tetrahedron Lett. 1975, 31, 2647-2650.

(2) Newkome, G. R.; Yao, Z; Baker, G. R.; Gupta, V. K. J. Org. Chem. 1985, 50, 2003-2004. 